\title{
Analysis of Small Amounts of D-Amino Acids and the Study of Their Physiological Functions in Mammals
}

\author{
Kenji Hamase, ${ }^{\dagger}$ Akiko Morikawa, Sachise Etoh, Yosuke ToJo, Yurika Miyoshi, and \\ Kiyoshi ZAITSU
}

Graduate School of Pharmaceutical Sciences, Kyushu University, 3-1-1 Maidashi, Higashi, Fukuoka 812-8582, Japan

\begin{abstract}
D-Amino acids, the enantiomers of L-amino acids, are candidates to be novel physiologically active substances and the biomarkers in mammals. However, the amounts of D-amino acids in the tissues and physiological fluids are extremely small in most cases, and sensitive and selective analytical methods are needed for their determination. In the present manuscript, we review the analytical technologies including our recent advances for the determinations of small amounts of D-amino acids in mammals and the applications to clarify their physiological meanings.
\end{abstract}

(Received February 28, 2009; Accepted May 11, 2009; Published August 10, 2009)

\begin{tabular}{|c|c|c|c|}
\hline 1 Introduction & 961 & 5 Day-Night Changes of D-Alanine and Related & \\
\hline 2 Two-dimensional HPLC Determination of Small & & Compounds & 966 \\
\hline Amounts of D-Amino Acids in Mammalian & & 6 Simultaneous Two-dimensional Determination of & \\
\hline Tissues and Physiological Fluids & 961 & Multiple D-Amino Acids in Mammals & 967 \\
\hline 3 Tissue Distribution of D-Alanine in Rodents & 962 & 7 Conclusion & 967 \\
\hline 4 Cellular Localization of D-Alanine in the Rat & & 8 Acknowledgements & 968 \\
\hline Pancreas and Pituitary Gland & 964 & 9 References & 968 \\
\hline
\end{tabular}

\section{Introduction}

D-Amino acids are the enantiomers of L-amino acids, and it has been long believed that only L-enantiomers are present in higher animals. However, with the recent advances in analytical techniques, several D-amino acids were found in mammals including human beings. ${ }^{1-3}$ Especially, D-aspartic acid (D-Asp) and D-serine (D-Ser) have been widely investigated. In 1986, Dunlop et al. first reported that relatively large amounts of D-Asp were present in the pituitary gland of rats; ${ }^{4}$ now, this D-amino acid is known to regulate hormonal synthesis and secretion in various endocrine glands. ${ }^{5,6}$ Concerning D-Ser, a large amount of the D-form (about half that of the L-form) was found in the rat brain in $1992,{ }^{7}$ and now this D-amino acid is recognized as an endogenous neuromodulator. ${ }^{8-11}$ Based on these outstanding studies, D-amino acids are now expected to be novel physiologically active substances and biomarkers. However, the amounts of D-amino acids in mammalian tissues are extremely small in most cases, and the determination is usually interfered with by large amounts of the L-enantiomers, peptides and amino compounds. Therefore, for the determination of these small amounts of D-amino acids, a highly sensitive and selective analytical method is needed. In the present review, we

† To whom correspondence should be addressed.

E-mail: hamase@phar.kyushu-u.ac.jp would like to focus on the advances in analytical technologies, including our recent publications, for the determination of small amounts of D-amino acids in mammalian tissues and physiological fluids. The application results for clarifying their physiological functions are also discussed.

\section{Two-dimensional HPLC Determination of Small Amounts of D-Amino Acids in Mammalian Tissues and Physiological Fluids}

The determination of amino acid enantiomers is a widely investigated topic in analytical chemistry; until now, a large number of analytical methods using GC, HPLC, HPCE and specific enzymes has been reported. ${ }^{1,12-16}$ Most of these methods have been established for the simple and rapid determination of various amino acid enantiomers, and these methods are useful for analyzing relatively large amounts of D-amino acids, such as D-Ser and D-Asp, in mammalian tissues. As described above, a large amount of D-Ser ( $c a .300 \mathrm{nmol} / \mathrm{g}$ tissue) is observed in the frontal brain, ${ }^{7,17-19}$ and this D-amino acid is now recognized as an endogenous neuromodulator. ${ }^{8-10}$ D-Asp is widely present in the endocrine tissues (higher than $100 \mathrm{nmol} / \mathrm{g}$ tissue) $)^{18,20,21}$ and its physiological function in regulating hormonal synthesis and secretion has gradually been clarified., ${ }^{5,22-28}$ However, the determination of small amounts of D-amino acids in mammalian tissues and physiological fluids has always been interfered with 
by thousands or more peptides, amino acids and other amino compounds. Therefore, a highly selective analytical method is essential.

For the sensitive and selective analysis of small amounts of D-amino acids in mammals, we have established two-dimensional HPLC (2D-HPLC) methods combining a microbore-reversed phase column and an enantioselective column. ${ }^{29-36}$ For the sensitive determination, amino acids are precolumn derivatized with 4-fluoro-7-nitro-2,1,3-benzoxadiazole ${ }^{37}$ (NBD-F, Fig. 1). NBD-F is a useful fluorescence derivatization reagent, and the derivatives, NBD-amino acids, which have a strong fluorescence at $530 \mathrm{~nm}$ with the excitation at $470 \mathrm{~nm}$ were determined by the 2D-HPLC system. Figure 2 shows a typical flow diagram of the 2D-HPLC system with a microbore-ODS column as the first dimension, and an enantioselective column as the second dimension. In the first dimension, the NBD-amino acids were separated as their D+L mixtures. Elution of the NBD-amino acids were monitored by a fluorescence detector. Only the fraction containing the target amino acid was collected in a loop, and then introduced into the enantioselective column in which the D- and L-enantiomers were separated. By using a microbore-ODS column for the first dimension, the volume of the amino acid fraction decreased and the entire volume of the fraction could be introduced into the enantioselective column without any loss of sensitivity. The 2D-HPLC chromatograms of the Ala enantiomers obtained using the rat cerebrum ${ }^{31}$ are indicated in Fig. 3. In this case, the amount of D-Ala is about $0.5 \%$ that of L-Ala, however, the small amount of the D-form could be successfully determined with minimal interference from the intrinsic substances in the brain tissue.

We have already reported the 2D-HPLC procedures for D-Ala, ${ }^{31}$ D-Leu, ${ }^{29,30}$ D-Ser, ${ }^{36}$ D-Pro analogs ${ }^{30,33,35}$ and branchedaliphatic D-amino acids. ${ }^{34}$ The values of lower limit of quantitation of D-amino acids using these methods are $500 \mathrm{amol}$ -

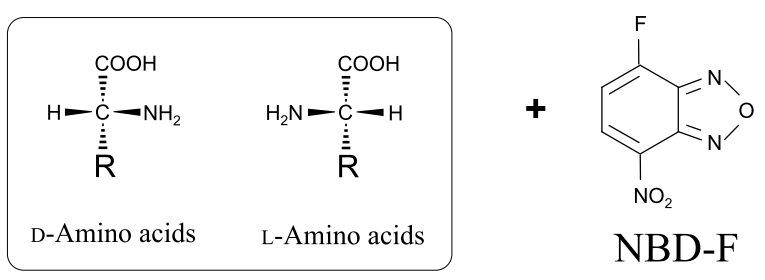

Fig. 1 Structures of D- and L-amino acids and NBD-F.
$5 \mathrm{fmol}$, and the RSD values for within- and between-day precisions are approximately $5 \%$ or lower. These results indicate that the 2D-HPLC procedures are powerful tools for analyzing small amounts of D-amino acids in mammalian tissues and physiological fluids.

\section{Tissue Distribution of D-Alanine in Rodents}

For clarification of the physiological meanings of D-amino acids in mammals, the investigations of tissue distributions often provide significant insights into their functions. D-Ser is reported to be widely present in the central nervous system. ${ }^{7,17-19}$ Especially, large amounts of D-Ser were observed in the frontal brain areas, such as the cerebrum, hippocampus, striatum and olfactory lobe $(100-300 \mathrm{nmol} / \mathrm{g}$ tissue $)$. On the other hand, the amounts in the cerebellum, medulla oblongata and spinal cord are extremely low. The tissue localization of D-Ser is closely related to the distribution of the $N$-methyl-D-aspartate (NMDA) subtype of the glutamate receptor in the brain. ${ }^{38,39}$ This D-amino acid is now well-known to modulate neurotransmission in the mammalian central nervous system..$^{8-10}$ Concerning D-Asp, relatively large amounts of this D-amino acid were found in the endocrine and neuro-endocrine tissues. ${ }^{18,20,21}$ For example, about
(A) RP-separation

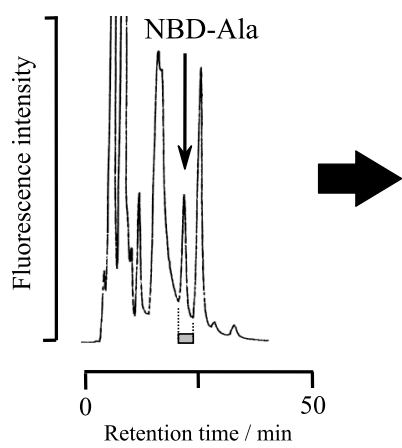

(B) Enantiomer separation

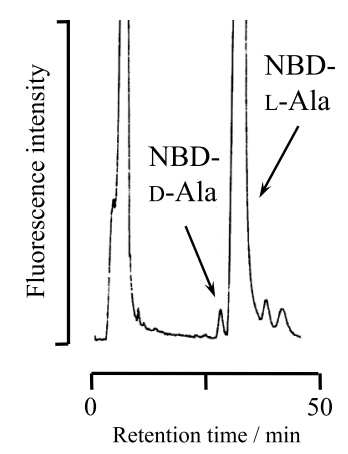

Fig. 3 Determination of D-Ala in the rat cerebrum. ${ }^{31}$ (A) Reversedphase separation of NBD-Ala in the first dimension, and (B) the interlinked enantiomer separation of NBD-D-Ala and NBD-L-Ala. The fraction indicated by the gray bar was on-line collected in a loop and then transferred to the enantioselective column.

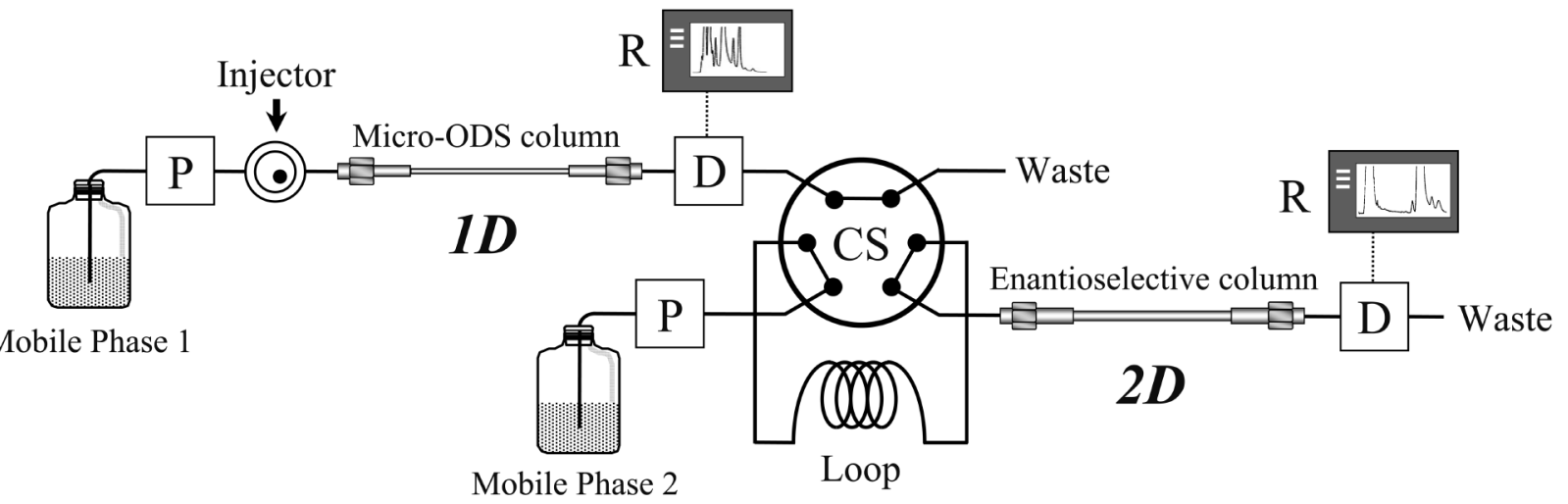

Fig. 2 Flow diagram of the 2D-HPLC system for the determination of D- and L-amino acids. P, Pump; $\mathrm{D}$, detector; R, integrator; CS, column-switching unit. 
(A) D-Ala, Rat

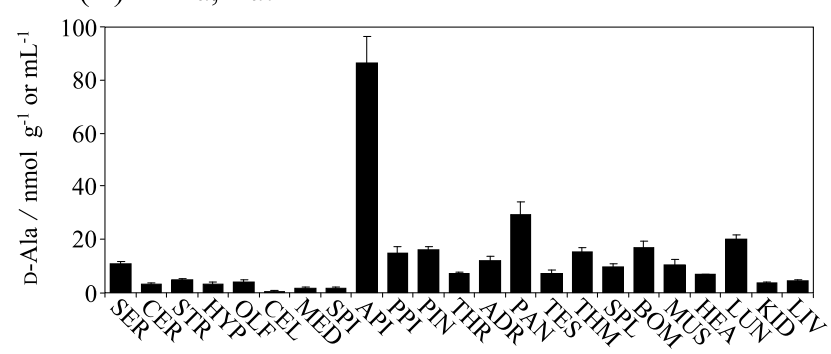

(B) D-Ala, Mouse

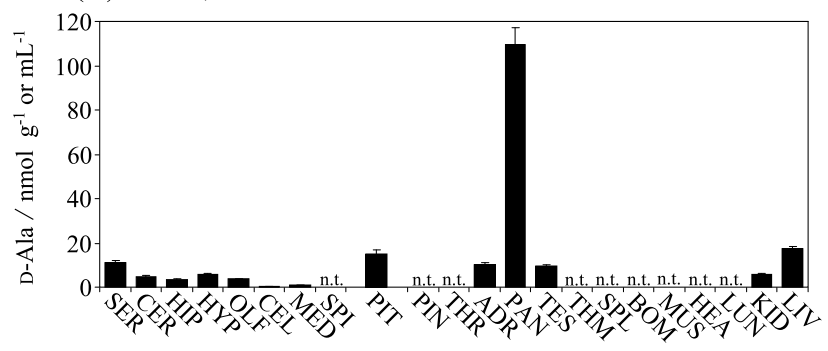

(C) D-Ser, Mouse

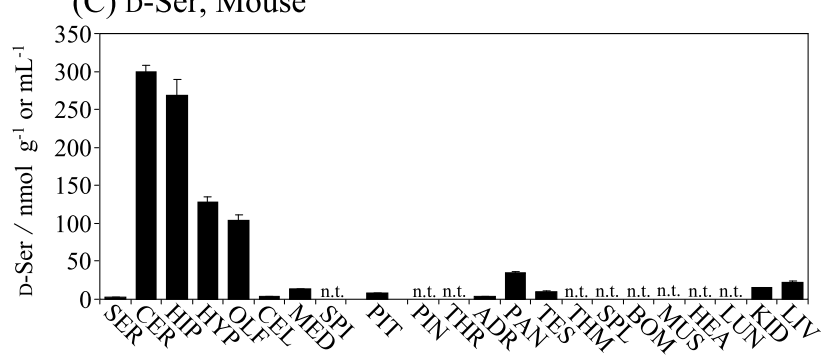

Fig. 4 Tissue distribution of D-Ala and D-Ser in rats and mice. ${ }^{31,36}$ (A) Male Wistar rats ( 9 weeks of age, SPF, $n=5$ ) were used. (B) and (C) Male ddY/DAO ${ }^{+}$mice ( 8 - 9 weeks of age, SPF, $n=3-5$ ) were used. SER, Serum; CER, cerebrum; STR, corpus striatum; HIP, hippocampus; HYP, hypothalamus; OLF, olfactory lobe; CEL, cerebellum; MED, medulla oblongata; SPI, spinal cord; API, anterior pituitary; PPI, posterior pituitary; PIT, pituitary gland; PIN, pineal gland; THR, thyroid gland; ADR, adrenal gland; PAN, pancreas; TES, testis; THM, thymus; SPL, spleen; BOM, bone marrow; MUS, muscle; HEA, heart; LUN, lung; KID, kidney; LIV, liver. Values represent mean \pm SE; n.t., not tested.

half of the Asp present in the rat pineal gland is D-form (higher than $1000 \mathrm{nmol} / \mathrm{g}$ tissue), ${ }^{21}$ and is reported to inhibit melatonin synthesis in the gland. ${ }^{40}$ Large amounts of D-Asp are also found in the testis, pituitary gland and adrenal gland, and the material is reported to regulate the synthesis and/or secretion of hormones such as testosterone and prolactin in the tissues. ${ }^{5,6,22-28,40}$

$\mathrm{D}$-Ala is one of the D-amino acids present in small amounts in mammalian tissues; its physiological function has not yet been clarified. To obtain fundamental knowledge of its function, we have investigated the tissue distribution of D-Ala in the brain and periphery of rodents. ${ }^{18,19,31,36,41}$ Figure 4(A) shows the distribution of D-Ala in the entire body of rats (22 tissues and serum). Among all the tested tissues, the highest amount of D-Ala was observed in the anterior pituitary gland, and the second highest amount was observed in the pancreas. ${ }^{31}$ In the rat anterior pituitary gland, the D-Ala amount was found to be $86.4 \mathrm{nmol} / \mathrm{g}$ tissue, which is 5.9 times higher than that in the posterior pituitary gland $(14.7 \mathrm{nmol} / \mathrm{g})$. However, concerning the proportion of the $\mathrm{D}$-form $(\mathrm{D} /(\mathrm{D}+\mathrm{L}) \times 100)$, the values are
(A) $\mathrm{OA}-2500 \mathrm{~S}$

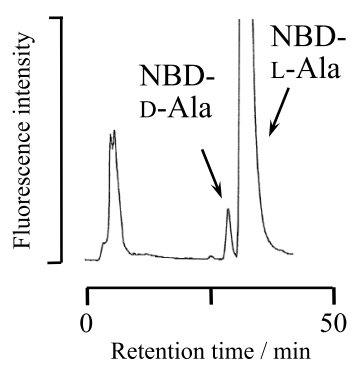

(B) OA-2500(R)

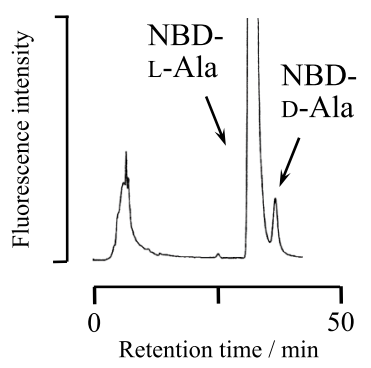

Fig. 5 Chromatograms of NBD-D-Ala and NBD-L-Ala in the rat pancreas obtained using (A) Sumichiral OA-2500S and (B) Sumichiral $\mathrm{OA}-2500(\mathrm{R})$ columns in the second dimension.

$2.5 \%$ for the anterior pituitary gland and $1.3 \%$ for the pancreas, indicating that the amounts of D-Ala are still extremely small in these tissues. Because the amounts of D-Ala in the tissues are extremely low, the presence and the determined values should be definitely confirmed. For that purpose, we used an opposite enantioselective column in the second dimension of the 2D-HPLC system described in Section 2. An example using the rat pancreas is demonstrated in Fig. 5. Figure 5(A) shows the chromatogram obtained using a Sumichiral OA-2500S column in the second dimension, in which D-Ala eluted ahead of L-Ala. On the other hand, using a Sumichiral OA-2500(R) column as the enantioselective column, the elution order of the enantiomers are reversed, and D-Ala eluted after L-Ala. By using both enantioselective columns, the retention times for D-Ala (the peak observed at the retention time of NBD-D-Ala in the tissues) were completely the same as those for the authentic D-Ala. In addition, the D-Ala amounts determined by the two enantioselective columns were almost equal, indicating that the peak observed at the retention time of NBD-D-Ala should be derived from the actual D-Ala and that no co-eluting interfering substances are present. The D-Ala amounts for all tissues were confirmed by the same procedure.

Figure 4(B) shows the tissue distribution of D-Ala in the mice. ${ }^{36}$ Similar to the results obtained for the rats, the amounts of D-Ala in the brain tissues are extremely low. Among all the tissues tested (12 tissues), the highest amount of D-Ala was observed in the pancreas. Concerning the mouse pituitary gland, the anterior pituitary gland and posterior pituitary gland are too small to accurately measure the tissue weights, thus the anterior and posterior lobes were not separated and were excised together as the pituitary gland. Although the amount is not so high as observed in the rat pituitary gland, a higher amount of D-Ala was present compared to those in the brain tissues. Figure $4(\mathrm{C})$ shows the distribution of D-Ser in the mice. ${ }^{36}$ D-Ser and D-Ala were reported to have similar functions in vitro to regulate the NMDA mediated neurotransmission..$^{9,42,43}$ However, the tissue distribution of these two D-amino acids are totally different; $\mathrm{D}$-Ser is localized in the frontal brain areas, such as the cerebrum and hippocampus, while D-Ala is localized in the anterior pituitary gland and in the pancreas. These results strongly suggest that the physiological function of these two D-amino acids are different in mammals. Further clarification of the physiological meanings of D-Ala is highly recommended. 


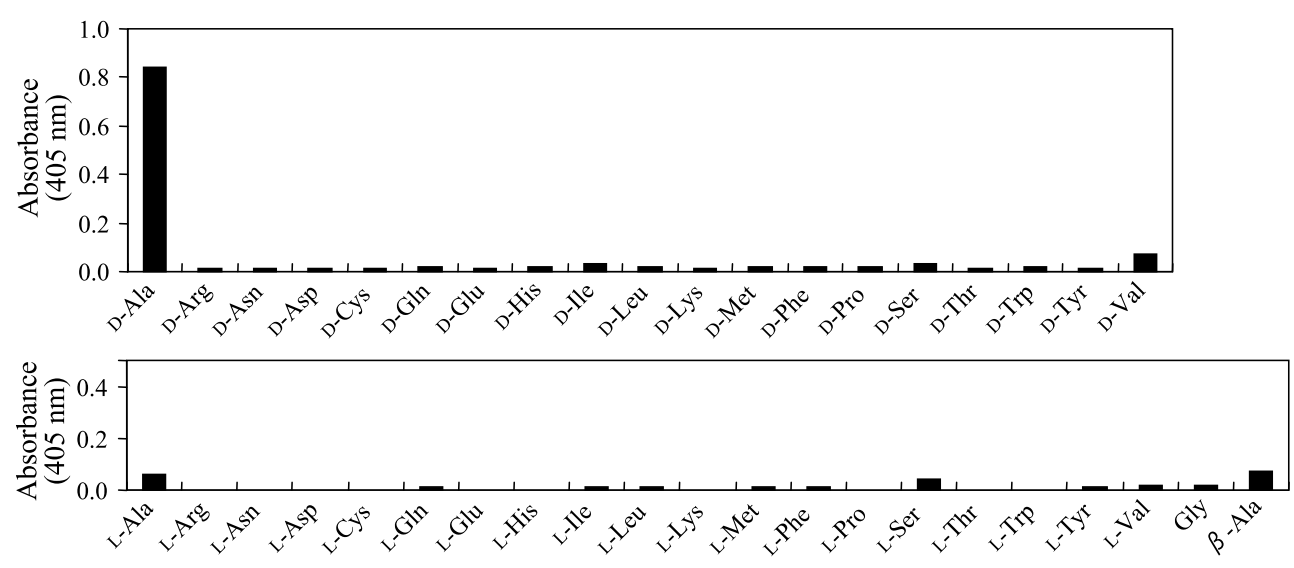

Fig. 6 Specificity of the mouse monoclonal antibody IgG against D-Ala. ${ }^{44}$ Values are the means of two analyses.
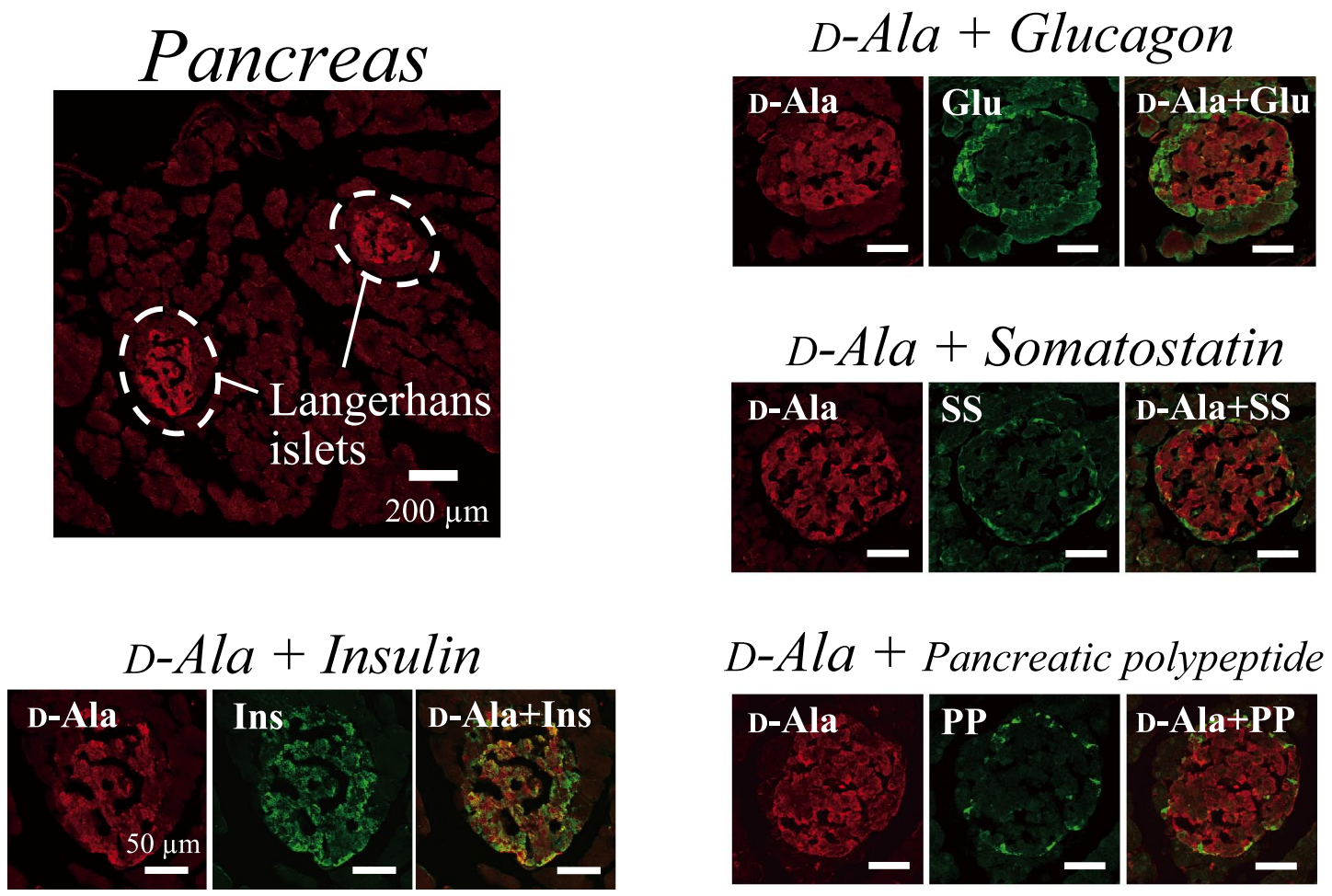

Fig. 7 Immunofluorescence staining of D-Ala and the double staining of D-Ala and pancreatic hormones in the rat pancreas. ${ }^{44} \mathrm{D}$-Ala is indicated in red, and the pancreatic hormones are indicated in green. Co-localization of D-Ala and the pancreatic hormone is indicated in yellow. Ins, Insulin; Glu, glucagon; SS, somatostatin; PP, pancreatic polypeptide.

\section{Cellular Localization of D-Alanine in the Rat Pancreas and Pituitary Gland}

As described in Section 3, D-Ala is localized in the pancreas and the anterior pituitary gland. ${ }^{31}$ These two tissues are well-known endocrine tissues containing various types of cells. The pancreas contains 4 types of endocrine cells secreting insulin, glucagon, somatostatin and pancreatic polypeptide, and other types of cells secreting digestive enzymes. The anterior pituitary gland contains 5 types of cells secreting growth hormone adrenocorticotropic hormone (ACTH), gonadotropic hormone, prolactin and thyroid-stimulating hormone. To perform the immunohistochemical staining of D-Ala in these tissues for clarifying the cellular localization of this D-amino acid, we raised a mouse monoclonal antibody against D-Ala. ${ }^{44}$ Figure 6 shows the selectivity of the obtained monoclonal antibody, indicating that the antibody specifically recognizes the D-Ala moiety with no significant cross reactivity to any other proteinogenic amino acid enantiomer. Using the obtained monoclonal antibody, we investigated the cellular localization of D-Ala in the pancreas ${ }^{44}$ and in the anterior pituitary gland. ${ }^{45}$ Figure 7 shows the immunofluorescence staining results of D-Ala in the rat pancreas. In the pancreas, D-Ala was specifically observed in the Langerhans islets. Considering that the volume of the Langerhans islets is only $1-2 \%$ of the pancreas, the 

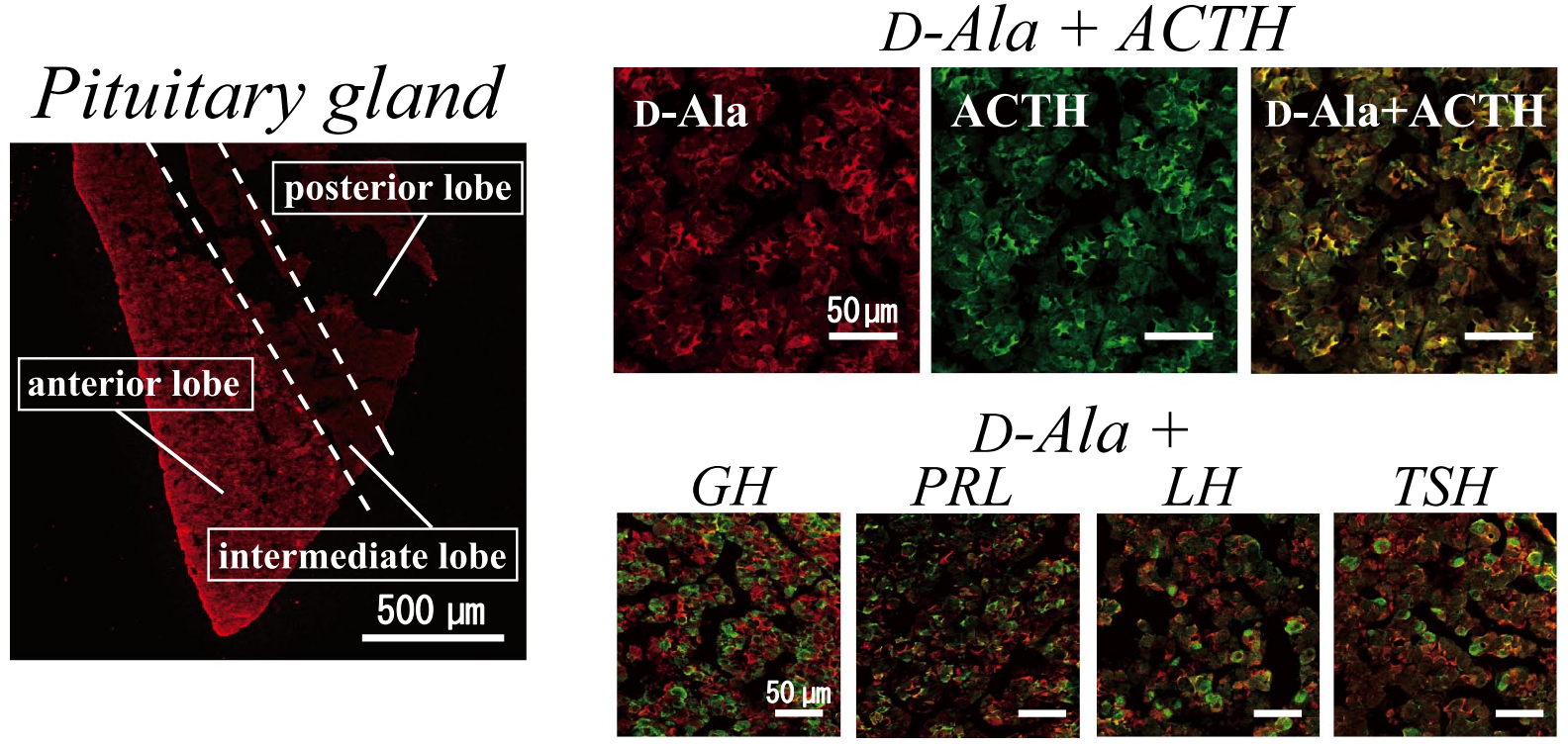

Fig. 8 Immunofluorescence staining of D-Ala and the double staining of D-Ala and pituitary hormones in the rat pituitary gland. ${ }^{45} \mathrm{D}$-Ala is indicated in red, and the pituitary hormones are indicated in green. Co-localization of D-Ala and the pituitary hormones is indicated in yellow. ACTH, Adrenocorticotropic hormone; GH, growth hormone; PRL, prolactin; LH, luteinizing hormone; TSH, thyroid-stimulating hormone.

(A) D-Ala

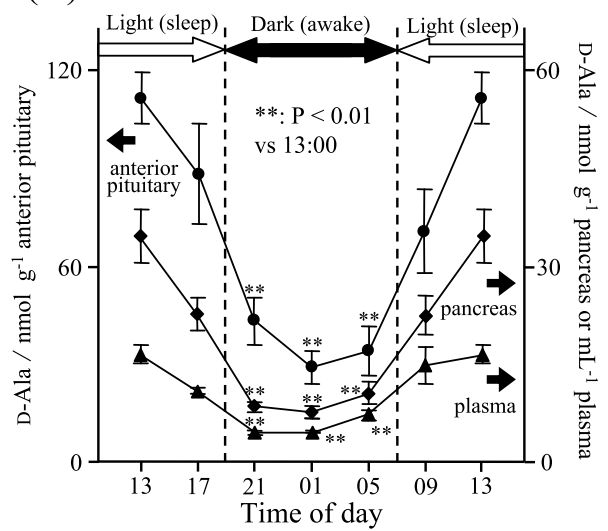

(C) D-Asp and D-Ser

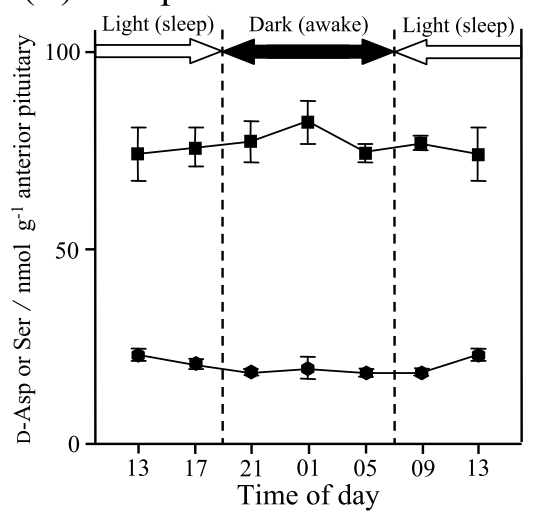

(B) L-Ala

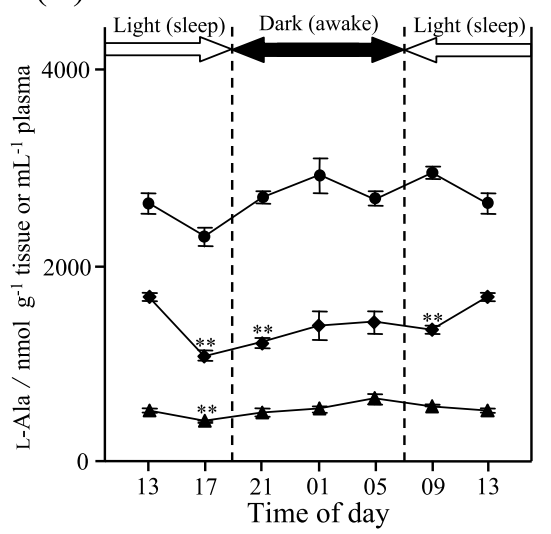

(D) L-Asp and L-Ser

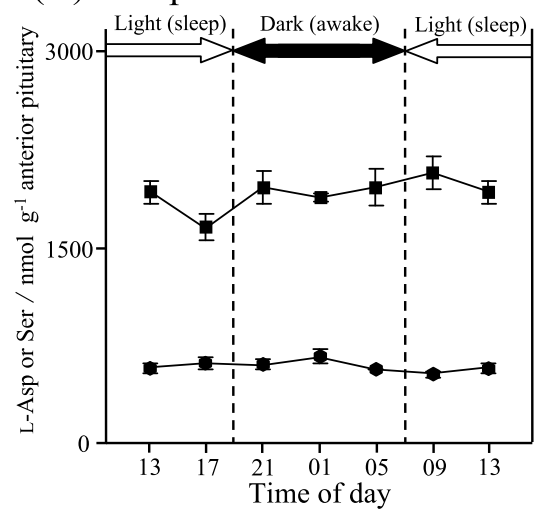

Fig. 9 Circadian changes of (A) D-Ala and (B) L-Ala amounts in the anterior pituitary gland (closed circles), pancreas (closed diamonds) and plasma (closed triangles). ${ }^{46}$ (C) and (D) Circadian changes of D- and L-Asp (closed squares) and D- and L-Ser (closed hexagons) in the rat anterior pituitary gland. The values represent mean \pm SE of 3 animals, and the asterisks indicate a significant decrease $(p<0.01)$ from the values at 13:00. 
concentration of D-Ala in the Langerhans islets is expected to be extremely high $(1000 \mathrm{nmol} / \mathrm{g}$ or higher). The double staining results of D-Ala and the pancreatic hormones clearly indicated that D-Ala is specifically localized in the insulin secreting $\beta$-cells. The immunofluorescence staining results of $\mathrm{D}$-Ala in the rat pituitary gland are shown in Fig. 8. A strong immunoreactivity was observed only in the anterior lobe, and no significant staining was observed in the intermediate and posterior lobes. These results are quite-consistent with the results obtained by the 2D-HPLC system. ${ }^{31}$ In the anterior pituitary gland, D-Ala was specifically localized in the ACTH secreting cells. As both ACTH and insulin are the well-known hormones regulating the blood-glucose level, D-Ala might have some function in regulating the blood-glucose level in mammals by regulating these hormones.

\section{Day-Night Changes of D-Alanine and Related Compounds}

During our study on clarifying the physiological function of D-Ala in mammals, we found that D-Ala shows a clear circadian rhythm. ${ }^{31,46}$ Figure 9 shows the day-night changes of D-Ala in the rat anterior pituitary gland, pancreas and plasma. In both the tissues and plasma, higher amounts of D-Ala were observed in the daytime than those observed in the nighttime. On the other hand, the amounts of L-Ala in these tissues and plasma do

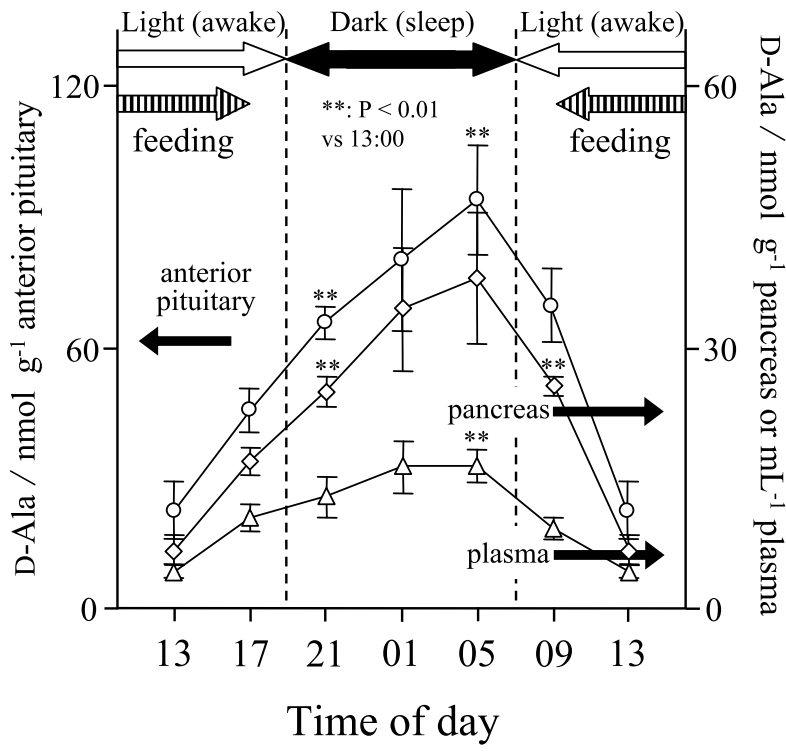

Fig. 10 Circadian changes of D-Ala amounts in the anterior pituitary gland (open circles), pancreas (open diamonds) and plasma (open triangles) under restricted feeding conditions. ${ }^{46}$ The values represent mean \pm SE of 3 animals, and the asterisks indicate a significant increase $(p<0.01)$ from the values at 13:00.

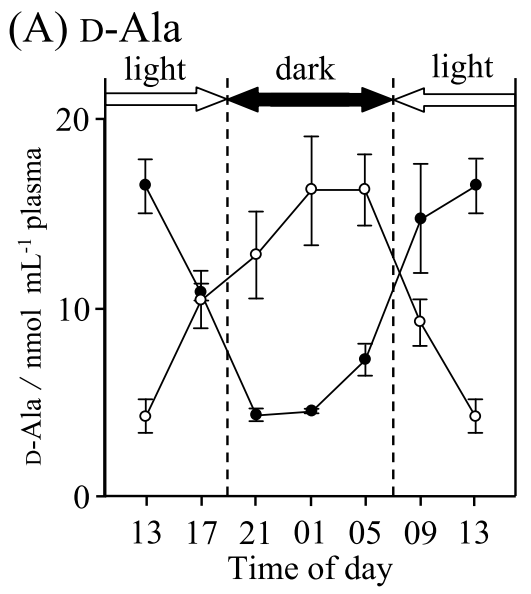

(C) Corticosterone

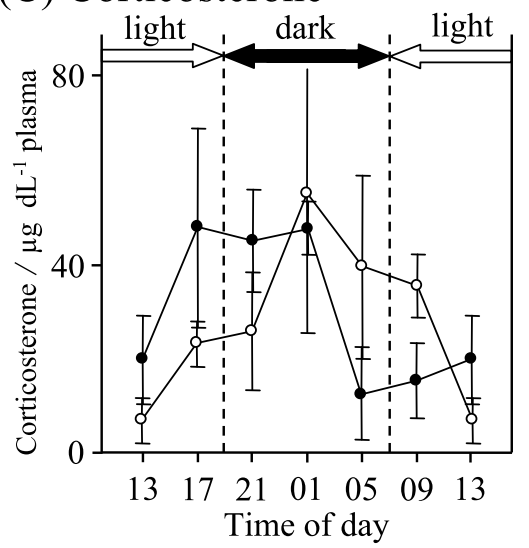

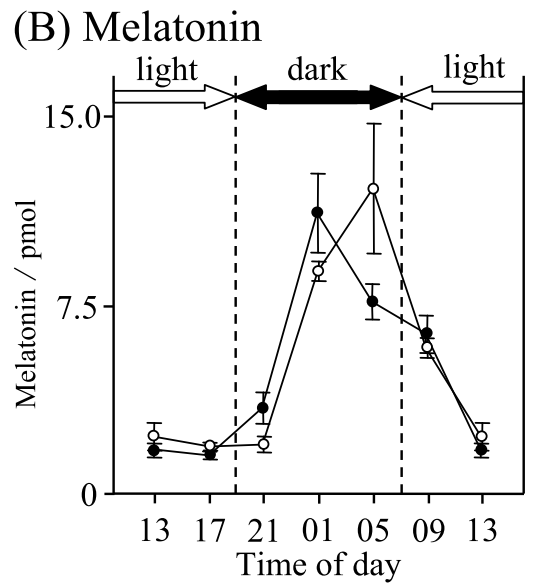

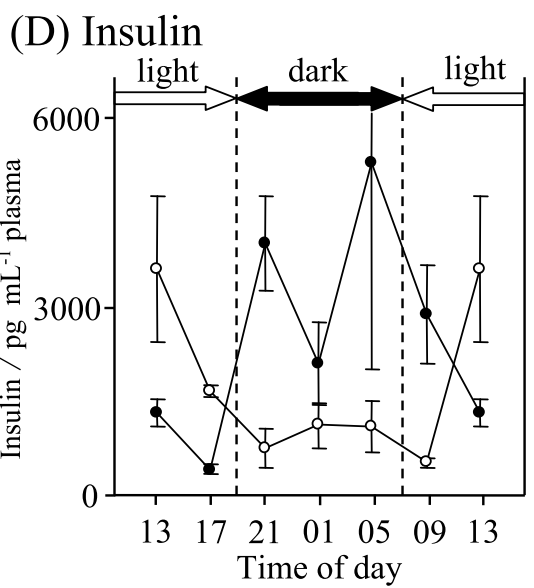

Fig. 11 Circadian changes of (A) D-Ala, (B) melatonin, (C) corticosterone and (D) insulin. ${ }^{46}$ Closed symbols indicate the values for the normal rats and open symbols indicate those of the food restricted rats. The values represent mean \pm SE of 3 animals. 


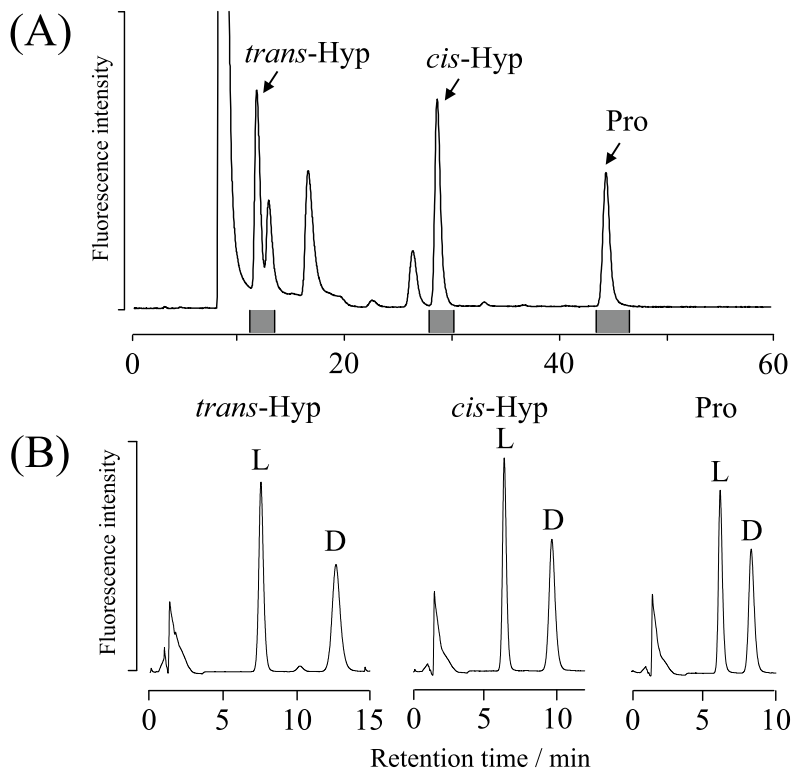

Fig. 12 2D-HPLC separation of Pro and Hyp enantiomers. ${ }^{35}$ (A) Reversed-phase separation of NBD-trans-Hyp, NBD-cis-Hyp and NBD-Pro in the first dimension, and (B) the interlinked enantiomer separations using a Chiralpak QN-2-AX column. The fractions indicated by gray bars were on-line collected in the loop device and successively transferred to the enantioselective column. Injection amounts of all enantiomers were $200 \mathrm{fmol}$.

not show clear circadian changes. The pituitary gland is also reported to contain D-Asp and D-Ser. ${ }^{18}$ Therefore, we investigated the day-night changes of Asp and Ser enantiomers in the anterior pituitary gland. ${ }^{46}$ However, as shown in Figs. 9(C) and 9(D), the amounts of D,L-Asp and D,L-Ser do not show significant day-night changes. These results indicate that the amount of D-Ala is regulated in a manner different from those for D-Asp and D-Ser. To clarify the mechanism controlling the D-Ala levels, we investigated the effect of activity rhythm on the amounts of D-Ala in the anterior pituitary gland, pancreas and in the plasma. ${ }^{46}$ Rats are animals with nocturnal habits in the wild, and the amounts of D-Ala are higher during their sleeping period (daytime). However, in the rats with diurnal habits made by restricted feeding during the daytime (these rats could access food only from 9:00 a.m. to 17:00 p.m.), the day-night profiles of D-Ala were totally reversed. As indicated in Fig. 10, the amounts of D-Ala are high during the nighttime (sleeping period for these rats), while the amounts are low during the daytime. Figure 11 shows the day-night changes of various hormones in the rats with diurnal and nocturnal habits. Although a clear reversal of the circadian profile of D-Ala in the plasma was observed, the circadian profiles of melatonin and corticosterone did not show significant phase shift. On the other hand, the circadian profile of insulin showed a distinct reversal by the reversal of the activity rhythm of the rats. These results strongly suggest that D-Ala might have a physiological function related to insulin, and further investigations are highly recommended.

\section{Simultaneous Two-dimensional Determination of Multiple D-Amino Acids in Mammals}

Because all of the D-amino acids are expected to be candidates for novel physiologically active substances and biomarkers, the
(A)

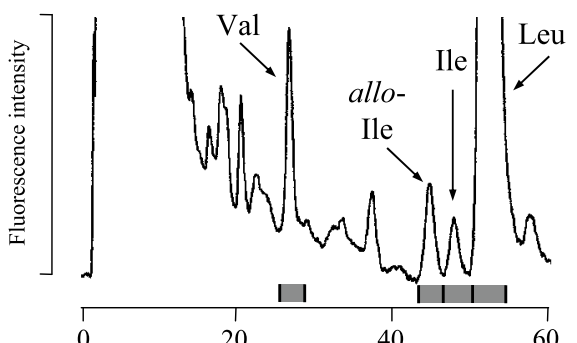

(B)

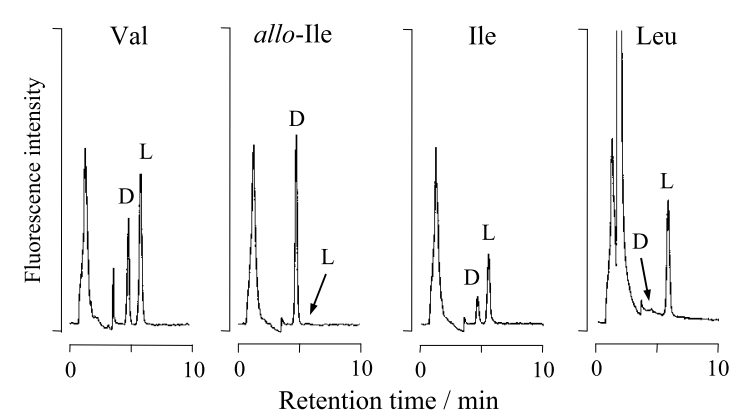

Fig. 13 2D-HPLC separation of branched aliphatic amino acid enantiomers in rat urine..$^{34}$ (A) Reversed-phase separation of NBD-Val, NBD-allo-Ile, NBD-Ile and NBD-Leu in the first dimension, and (B) the interlinked enantiomer separations using a Chiralpak QN-1-AX column. The fractions indicated by gray bars were on-line collected in the loop device and successively transferred to the enantioselective column.

simultaneous determination of multiple D-amino acids without the loss of selectivity and sensitivity is needed. For this purpose, we have established fully automated 2D-HPLC systems employing a microbore-ODS column and an enantioselective column. Until now, 2D-HPLC procedures for branched aliphatic amino acids (Val, allo-Ile, Ile and Leu) ${ }^{34}$ and Pro analogs (Pro, trans-Hyp and cis-Hyp) ${ }^{35}$ have been reported. The typical 2D-HPLC chromatograms are shown in Fig. 12. In the first dimension, NBD-amino acids were separated by a micro-ODS column as their $\mathrm{D}+\mathrm{L}$ mixtures. The fractions were on-line collected in the loop device, and then successively transferred to the enantioselective column. In the second dimension, a Chiralpak QN-1-AX column was used for the enantioseparation of the branched aliphatic amino acids, and a Chiralpak QN-2-AX column was used for the enantioseparation of the Pro analogs. All of these enantiomers were nicely separated in about $10 \mathrm{~min}$ $\left(1.22<\alpha<1.83,2.72<R_{\mathrm{s}}<6.17\right)$. Figure 13 shows the enantiomer determination of the branched aliphatic amino acids in rat urine. In the rat urine, relatively large amounts of D-Val, D-allo-Ile and D-Ile, and a small amount of D-Leu were found. These results indicate that various D-amino acids are actually present in mammals, and more studies to clarify the presence and distribution of all D-amino acids are currently in progress.

\section{Conclusion}

In the present review, we have summarized the analytical methods for the determination of small amounts of D-amino acids in mammalian tissues and physiological fluids, and the recent efforts to clarify their physiological meanings. In addition to widely reported D-Ser and D-Asp, various small amounts of D-amino acids are present in mammals. In particular, D-Ala is specifically localized in the cells closely related to the regulation 
of the blood-glucose level. Further studies to clarify their physiological meanings and the diagnostic values are highly recommended.

\section{Acknowledgements}

This work was supported in part by a Grant-in-Aid for Scientific Research from the Ministry of Education, Culture, Sports, Science and Technology of Japan, by the Takeda Science Foundation, and by an Industrial Technology Research Grant Program in '05 from New Energy and Industrial Technology Development Organization (NEDO) of Japan. The authors appreciate Shiseido Co., Ltd., (Tokyo, Japan) for providing the HPLC system (NANOSPACE series) and their technical support.

\section{References}

1. K. Hamase, A. Morikawa, and K. Zaitsu, J. Chromatogr., $B, \mathbf{2 0 0 2}, 781,73$.

2. N. Fujii, Orig. Life Evol. Biosph., 2002, 32, 103.

3. R. Konno, H. Brückner, A. D'Aniello, G. Fisher, N. Fujii, and H. Homma (ed.), "D-Amino Acids: A New Frontier in Amino Acids and Protein Research-Practical Methods and Protocols", 2007, Nova Science Publishers, New York.

4. D. S. Dunlop, A. Neidle, D. McHale, D. M. Dunlop, and A. Lajtha, Biochem. Biophys. Res. Commun., 1986, 141, 27.

5. H. Homma, Viva Origino, 2002, 30, 204.

6. H. Wolosker, A. D'Aniello, and S. H. Snyder, Neuroscience, 2000, 100, 183.

7. A. Hashimoto, T. Nishikawa, T. Hayashi, N. Fujii, K. Harada, T. Oka, and K. Takahashi, FEBS Lett., 1992, 296, 33.

8. T. Nishikawa, Biol. Pharm. Bull., 2005, 28, 1561.

9. S. H. Snyder and P. M. Kim, Neurochem. Res., 2000, 25, 553.

10. H. Wolosker, S. Blackshaw, and S. H. Snyder, Proc. Natl. Acad. Sci. U. S. A., 1999, 96, 13409.

11. K. Miya, R. Inoue, Y. Tanaka, M. Abe, R. Natsume, K. Sakimura, K. Hongou, T. Miyawaki, and H. Mori, J. Comp. Neurol., 2008, 510, 641.

12. N. M. Maier, P. Franco, and W. Lindner, J. Chromatogr., A, 2001, 906, 3.

13. V. Schurig, J. Chromatogr., A, 2001, 906, 275.

14. I. Ilisz, R. Berkecz, and A. Péter, J. Pharm. Biomed. Anal., 2008, 47,1 .

15. H. Wan and L. G. Blomberg, J. Chromatogr., A, 2000, 875, 43.

16. L. Sánchez-Hernández, A. L. Crego, M. L. Marina, and C. García-Ruiz, Electrophoresis, 2008, 29, 237.

17. A. Hashimoto, T. Oka, and T. Nishikawa, Eur. J. Neurosci., 1995, 7, 1657.

18. K. Hamase, H. Homma, Y. Takigawa, T. Fukushima, T. Santa, and K. Imai, Biochim. Biophys. Acta, 1997, 1334, 214.

19. A. Morikawa, K. Hamase, T. Inoue, R. Konno, A. Niwa, and K. Zaitsu, J. Chromatogr., B, 2001, 757, 119.

20. A. Hashimoto, T. Nishikawa, T. Oka, T. Hayashi, and K.
Takahashi, FEBS Lett., 1993, 331, 4.

21. K. Imai, T. Fukushima, K. Hagiwara, and T. Santa, Biomed. Chromatogr., 1995, 9, 106.

22. A. D'Aniello, Brain Res. Rev., 2007, 53, 215.

23. J. A. Lee, H. Homma, K. Sakai, T. Fukushima, T. Santa, K. Tashiro, T. Iwatsubo, M. Yoshikawa, and K. Imai, Biochem. Biophys. Res. Commun., 1997, 231, 505.

24. S. Ishio, H. Yamada, M. Hayashi, S. Yatsushiro, T. Noumi, A. Yamaguchi, and Y. Moriyama, Neurosci. Lett., 1998, 249, 143.

25. G. D’Aniello, A. Tolino, A. D’Aniello, F. Errico, G. H. Fisher, and M. M. D. Fiore, Endocrinology, 2000, 141, 3862.

26. K. Sakai, H. Homma, J. A. Lee, T. Fukushima, T. Santa, K. Tashiro, T. Iwatsubo, and K. Imai, Biochem. Biophys. Res. Commun., 1997, 235, 433.

27. Y. Nagata, H. Homma, J. A. Lee, and K. Imai, FEBS Lett., 1999, 444, 160.

28. A. D’Aniello, A. D. Cosmo, C. D. Cristo, L. Annunziato, L. Petrucelli, and G. Fisher, Life Sci., 1996, 59, 97.

29. T. Inoue, K. Hamase, A. Morikawa, and K. Zaitsu, $J$. Chromatogr., B, 2000, 744, 213.

30. K. Hamase, T. Inoue, A. Morikawa, R. Konno, and K. Zaitsu, Anal. Biochem., 2001, 298, 253.

31. A. Morikawa, K. Hamase, and K. Zaitsu, Anal. Biochem., 2003, 312, 66 .

32. H. Zhao, K. Hamase, A. Morikawa, Z. Qiu, and K. Zaitsu, J. Chromatogr., B, 2004, 810, 245.

33. K. Hamase, S. Takagi, A. Morikawa, R. Konno, A. Niwa, and K. Zaitsu, Anal. Bioanal. Chem., 2006, 386, 705.

34. K. Hamase, A. Morikawa, T. Ohgusu, W. Lindner, and K. Zaitsu, J. Chromatogr., A, 2007, 1143, 105.

35. Y. Tojo, K. Hamase, M. Nakata, A. Morikawa, M. Mita, Y. Ashida, W. Lindner, and K. Zaitsu, J. Chromatogr., B, 2008, 875, 174.

36. Y. Miyoshi, K. Hamase, Y. Tojo, M. Mita, R. Konno, and K. Zaitsu, J. Chromatogr., B, in press.

37. K. Imai and Y. Watanabe, Anal. Chim. Acta, 1981, 130, 377.

38. A. Hashimoto, T. Nishikawa, T. Oka, and K. Takahashi, J. Neurochem., 1993, 60, 783.

39. M. J. Schell, M. E. Molliver, and S. H. Snyder, Proc. Natl. Acad. Sci. U. S. A., 1995, 92, 3948.

40. Y. Takigawa, H. Homma, J. A. Lee, T. Fukushima, T. Santa, T. Iwatsubo, and K. Imai, Biochem. Biophys. Res. Commun., 1998, 248, 641.

41. A. Morikawa, K. Hamase, T. Inoue, R. Konno, and K. Zaitsu, Amino Acids, 2007, 32, 13.

42. N. W. Kleckner and R. Dingledine, Science, 1988, 24, 835.

43. K. Sakata, T. Fukushima, L. Minje, T. Ohgusu, H. Taira, M. Mishina, and R. Shingai, Biochemistry, 1999, 38, 10099.

44. A. Morikawa, K. Hamase, T. Ohgusu, S. Etoh, H. Tanaka, I. Koshiishi, Y. Shoyama, and K. Zaitsu, Biochem. Biophys. Res. Commun., 2007, 355, 872.

45. S. Etoh, K. Hamase, A. Morikawa, T. Ohgusu, and K. Zaitsu, Anal. Bioanal. Chem., 2009, 393, 217.

46. A. Morikawa, K. Hamase, Y. Miyoshi, S. Koyanagi, S. Ohdo, and K. Zaitsu, J. Chromatogr., B, 2008, 875, 168. 